\title{
Self-assessment pain control in end-of-life children with bone tumors: a cohort study
}

\author{
Flavio Andrade ${ }^{1}$, Cristina Valete ${ }^{2}$, André Ricardo Silva ${ }^{3}$, and Sima Ferman ${ }^{4}$ \\ ${ }^{1} \mathrm{INCA}$ \\ ${ }^{2}$ Universidade Federal de São Carlos \\ ${ }^{3}$ Universidade Federal Fluminense Hospital Universitario Antonio Pedro \\ ${ }^{4}$ Instituto Nacional do Cancer
}

June 1,2021

\begin{abstract}
BACKGROUND: INCA estimated, for 2020 - 2022, Brazil would have 8,460 new pediatric cancer cases and 5\% are bone cancer. Pain is the most prevalent symptom and is present in $75 \%-90 \%$. Advanced ill patients have $40 \%$ pain undertreated. OBJECTIVE: This study was to evaluate pain in pediatric bone cancer patients and investigate if there was a difference between those who survived and those at end-of-life. PROCEDURE: Patients were registered at INCA Pediatric Department, January 2011 - October 2016, with the diagnosis confirmed of primary bone cancer and under 19 years old. Pain was evaluated and registered at three moments during their treatment: at registration, three months after and the last visit before the end of this study or patients death, using pain scores. RESULTS: 157 patients were bone cancer, 15 (9.6\%) had lost the follow up; 142 were analyzed, osteosarcoma $69.7 \%$ and Ewing sarcoma $30.3 \%$, metastatic patients $50.7 \%$. At the registration $53.5 \%$ had pain, $69.71 \%$ were receiving pain treatment and $42.25 \%$ had pain medications changed. Comparison of the three study moments was observed a decrease of pain status, with the absence of excruciating pain, and an increased use of opioids. There was no difference $(p=0.68)$ in pain status between groups of who survived and who died $(39.4 \%)$. CONCLUSION: Pain management resulted in reduction of pain complaint and reduction in pain intensity, together with increasing opioid use. End-of-life patients did not have more pain than others, but disease progression was associated to more pain.
\end{abstract}

\section{Introduction}

The Brazilian National Cancer Institute (INCA) estimated that cancer incidences in Brazil for 2020 - 2022 will be 685,960 new patients and 8,460 will be in children and adolescents ${ }^{1}$. Of this small population, $5 \%$ are bone cancers cases, osteosarcomas (OS) and Ewing sarcomas (ES) ${ }^{1,2}$. Pain is the symptom most prevalent in these types of cancer which is estimated to be present in about $75 \%$ to $90 \%$ of patients with advanced cancer $^{3-9}$ and can be present from the moment of the diagnosis to the end-of-life ${ }^{10-12}$.

With advances in pediatric bone cancer treatment, especially the use of intensive combination chemotherapies and supportive care, pediatric bone cancer death rates have been declining ${ }^{13}$. However, pain continues to be the most prevalent symptom in this population, and it has an important role in their quality of life ${ }^{14,15}$, especially in patients with advanced disease and in end-of-life period.

Although there are many pain management guidelines, as World Health Organization (WHO) and Expert Working Group of European Association for the Palliative Care. Pain undertreatment is still a problem and represent $40 \%$ of patients with advanced cancer ${ }^{16-18}$, one of the reasons for this untreated pain is the inappropriate use of opioids ${ }^{19}$.

This study had the objective to evaluate pain management in pediatric patients with bone tumors during 
treatment and follow up and to investigate if there was difference in pain status at last evaluation between those on treatment or in disease control and those at the end-of-life.

\section{Material and Methods}

This was a retrospective cohort study of pediatric patients with the diagnosis of primary bone cancer consecutively registered at the Pediatric Oncology Department of INCA, from January 12011 to October 16 2016. This study was approved by the INCA Research Ethics Committee (CAAE: 67729317.5.0000.5274).

Patients under 19 years old were included, they were registered at INCA's Pediatric Oncology Department with primary bone cancer, with either the diagnosis of OS or bone ES. Patients treated at other institutions and admitted at INCA for exclusive radiotherapy were excluded. The diagnosis, OS or ES, was confirmed by INCA Department of Pathology. Whenever the diagnosis had been carried out in other institutions, they had a pathology review at INCA. All patients were registered in Latin American Cooperative Group for Osteosarcoma Protocol of Treatment (GLATO) or in Latin American Cooperative Group for Ewing Tumors Family (GALLOP). ${ }^{12,20-23}$

At the time of the registration, all patients were initially evaluated for pain. If they were already using some kind of pain control medication for this symptom this was modified whenever necessary according to the patient's clinical evaluation. Pain management was performed by the multidisciplinary team according to institutional clinical protocols established by the pediatric pain clinic service.

In this study, pain measurement was standardized according to Wong and Baker, who had considered 0 as no pain; 1 to 3 as mild pain, 4 to 6 as moderate pain, 7 to 9 as severe pain and 10 as excruciating pain ${ }^{24-26}$.

Data was collected from medical records and registered in a specific chart. Three moments were considered during this cohort: at the registration moment, three months after registration and at the last visit before the end of this study or patients death. Patients were considered end-of-life when they had an evaluation up to 1 month before death ${ }^{27-29}$.

The variables of this study were: age at diagnosis, sex, race, cancer type, presence of metastases at diagnosis, progression disease, pain complaint, pain measurement, pain relieve treatment, modification in pain relief treatment and pain status at the end-of-life.

Statistical analysis was performed using SPSS for Windows (version 22) and Stata 13.0 (Stata Corp, LC ). The Kolmogorov-Smirnov Test was used to verify normality, results are presented as median, interquartile range (IQR) and 95\% confidence intervals $(95 \% \mathrm{CI})$. The association between categorical variables was tested by Pearson's Chi-squared test or Fisher exact test; Mann-Whitney test was used to compare continuous variables. Data was analyzed until December 31, 2016. We compared pain status in the last evaluation between those on treatment or in disease control and those at the end-of-life.

To identify possible associations between variables and pain status at the last evaluation, we performed a Poisson regression with robust estimation, log link function to determine crude prevalence ratios (CPR); patients were tested for characteristics, presence of metastasis at registration, disease progression at last evaluation and use of mild analgesics, weak opioids and strong opioids at last evaluation; variables associated to pain $(\mathrm{CPR}$ with $\mathrm{p}$-value $<0.20)$ were included in a multivariate model to determine adjusted prevalence ratios (APR). Considered a p-value of $<0.05$.

\section{Results}

During this cohort period, INCA Pediatric Oncology registered 1,625 patients, from these, 917 patients had the diagnosis of cancer, 157 were primary bone cancer who had been included.

Fifteen patients $(9.6 \%)$ were loss of some data, but these patients were analyzed: $9(60 \%)$ with diagnosis of OS and 6 (40\%) ES; median age 12 years old (IQR 8 - 14) and 4 (26.7\%) died. Comparisons were made between loss of data patients and the cohort sample according to age (Mann-Whitney Test; $\mathrm{p}=0.42$ ), diagnosis (Fisher Exact Test; $\mathrm{p}=0.30$ ) and survival (Fisher Exact Test; $\mathrm{p}=0.24$ ). 
The cohort study comprised 142 patients, with median age 13 years old (IQR 9-14 years old), 43.6\% male sex, being 99 OS (69.7\%) and $43 \mathrm{ES}(30.3 \%)$. Median time for initial treatment after registration was 22.5 days (IQR 16.0 - 34.0 days) and $72(50.7 \%)$ patients had metastatic disease at presentation, 46/99 OS (46.46\%) and 26/43 ES (60.46\%). Patients characteristics at registration are in Table 1.

We examined pain characteristics and previous treatment at registration. Seventy-six patients (53.5\%) had pain evaluated by a method at that moment and 99 (69.71\%) were in previous use of pain treatment.

At registration, pain treatment was modified in 60 patients $(42.25 \%)$; pain treatment at that moment evaluation were prescribed to 103 patients $(72.53 \%)$ in the following frequency: mild analgesics in $42(29.57 \%)$, weak opioids in $37(26.05 \%)$ and strong opioids in $17(11.97 \%)$, strong opioid plus mild analgesics in 1 $(0.70 \%)$, strong opioid plus mild analgesics in $6(4.22 \%)$, anticonvulsants in $11(7.75 \%)$, antidepressants in $21(14.79 \%)$ and steroids in $3(2.11 \%)$. Three patients with no pain had their pain treatment discontinued. It was initially observed a reduction in the use of mild analgesics and an increase in the use of weak and strong opioids.

Three months after registration, patients were evaluated again. Eight patients (5.63\%) had disease progression. Seventy-one patients $(50 \%)$ had their pain treatment changed. Pain treatment at that moment was: $21(14.78 \%)$ mild analgesics, $29(20.42 \%)$ mild opioids, $55(38.73 \%)$ strong opioids, $4(2.81 \%)$ mild and strong opioids, $2(1.40 \%)$ mild analgesics and weak opioid, $36(25.35 \%)$ anticonvulsants, $60(42.25 \%)$ antidepressants and $4(2.81 \%)$ steroids.

At last evaluation, all 142 patients had received antineoplastic treatment, 105 (73.94\%) were submitted to surgery, $31(21.83 \%)$ to radiotherapy and $62(43.66 \%)$ to chemotherapy. Sixty-five patients $(45.77 \%)$ had disease in progression. Seventy-three patients $(51.41 \%)$ had their pain treatment changed. Pain treatment at that last evaluation was: 19 (13.38\%) mild analgesics, 21 (14.78\%) mild opioids, 63 (44.36\%) only strong opioids, $3(2.11 \%)$ weak and strong opioid (Figure 1).

The comparison of pain at registration, three months later and at last evaluation showed along the time a more frequent pain assessment and absence of patients with excruciating pain (Table 2).

A hundred and twenty-two patients had pain classified at last evaluation. Twenty patients who did not have pain assessment by scale, reported no pain at last evaluation and were classified as no pain.

According to this, data was imputed based on pain complaint at last evaluation (self-assessment). Analysis between those who survived or died, revealed no difference of pain status between groups $(\mathrm{p}=0.68)$. Fiftysix patients evolved to death $(39.4 \%)$, it was observed a $25 \%(14 / 56)$ frequency of pain complaint among those end-of-life patients and a $22 \%(19 / 86)$ among the others at last evaluation (Table 3$)$.

Poisson regression to estimate CPR of pain (yes/no) at last evaluation revealed no difference of pain status between those end-of-life or not ( $\mathrm{p}=0.68)$, as can be seen on Table 4. All variables with CPR $\mathrm{p}$ value $<0.20$ were candidates to enter a multivariate model to predict adjusted prevalence ratios (APR).

A multivariate model to estimate APR included disease progression, use of strong opioids and steroids at last evaluation. This model was adjusted for age (Table 5).

\section{Discussion}

In the present study, there was a high incidence of metastatic disease at presentation (50.70\%). Pakos et al ${ }^{30}$, in an international OS compilation, where OS Brazilian Group was included, showed $13 \%$ of the patients with metastatic disease, the same could be seen in the OS Children Oncology Group (COG) (27\%) and Cooperative Ewing Sarcoma Study (CESS) group (30\%) ${ }^{30-34}$. Patients arrived at INCA with advanced disease and this characteristic probably influenced in their pain status in all follow-up moments.

At registration, pain was related as the most frequent symptom (61.27\%). Even if pain scales were not used, the majority of patients self-assessed pain as moderate to severe and three children had excruciating pain. At that moment, the most frequent drugs used for pain control were mild analgesics (paracetamol or dipyrone) 
and treatment was changed in $42.25 \%$ of patients. This data contrasts with the WHO recommendation, that suggested the use of strong opioids at that level of pain, specially morphine. This undertreatment could be attributed to the fear of using opioids ${ }^{16,35-38}$.

By contrast, end-of-life patients used strong opioids and morphine was the most frequently used, followed by anticonvulsant and antidepressant drugs, as well as other drugs and procedures, as a multimodal therapy. This has been used in agreement with international references. ${ }^{27,28,35,39}$

Along the follow-up period, more patients had their pain classified. Also, pain complaint decreased, and no excruciating pain was observed at last evaluation. At registration, $34.21 \%$ had no pain and at last evaluation $73.77 \%$ of all patients had no pain. These results suggest the effectiveness of the management of pain control associated during follow-up.

However, we observed association between pain at last evaluation and the presence of disease progression. Those patients presented 3.5 times more pain compared to the others. Comparing with previous international cooperative pediatric bone cancer groups studies, the influence of this variable followed the same direction, being associated to pain ${ }^{20-23,31-34,40-42}$.

At last evaluation we observed no difference in pain complaint between end-of-life patients and the others. This suggests that pain management was effective. Also, it was observed opioid use associated to pain complaint at last evaluation, this is a reverse cause-effect association, as those who have more pain use more opioids. However, $19.64 \%$ of end-of-life patients and $18.60 \%$ of those undergoing treatment or in disease control still presented moderate and severe pain, at last evaluation. We suggest that this might have occurred because of family misconception for the use of pain control drugs. Similar results were seen by Snaman et al., who revealed difficulty in pain control, with the principal obstacle being lack of information and misconceptions of opioid use. ${ }^{5,29,27,43}$.

\section{Conclusion}

In this study, pain management together with increasing opioid use in pediatric patients with bone tumors resulted in reduction of pain complaint and in pain intensity. End-of-life patients did not have more pain than the survivors, but disease progression was associated to more pain.

\section{Conflict of Interest}

The authors have no financial or other conflicts of interest relevant to this article.

\section{Referencies}

1. De Oliveira Santos M. Estimativa/2020 - Incidência de Câncer no Brasil. Rev Bras Cancerol . 2020;66(1). doi:10.32635/2176-9745.RBC.2020v66n1.927

2. Santos M de O. Estimativa 2018: Incidência de Câncer no Brasil.Rev Bras Cancerol . 2018;64(1):119-120. doi:10.32635/2176-9745.RBC.2018v64n1.115

3. Wiffen PJ, Derry S, Moore RA, et al. Oral paracetamol (acetaminophen) for cancer pain. Cochrane Pain, Palliative and Supportive Care Group, ed. Cochrane Database Syst Rev . Published online July 12, 2017. doi:10.1002/14651858.CD012637.pub2

4. Portenoy RK, Lesage P. Management of cancer pain. The Lancet . 1999;353(9165):1695-1700. doi:10.1016/S0140-6736(99)01310-0

5. van den Beuken-van Everdingen MHJ, Hochstenbach LMJ, Joosten EAJ, Tjan-Heijnen VCG, Janssen DJA. Update on Prevalence of Pain in Patients With Cancer: Systematic Review and Meta-Analysis. J Pain Symptom Manage . 2016;51(6):1070-1090.e9. doi:10.1016/j.jpainsymman.2015.12.340

6. Wiffen PJ, Wee B, Moore RA. Oral morphine for cancer pain. Cochrane Pain, Palliative and Supportive Care Group, ed. Cochrane Database Syst Rev . Published online April 22, 2016. doi:10.1002/14651858.CD003868.pub4 
7. Deng D, Fu L, Zhao YX, et al. The Relationship Between Cancer Pain and Quality of Life in Patients Newly Admitted to Wuhan Hospice Center of China. Am J Hosp Palliat Med . 2012;29(1):53-59. doi:10.1177/1049909111418636

8. Dreidi MM, Hamdan-Mansour AM. Pain, Sleep Disturbance, and Quality of Life Among Palestinian Patients Diagnosed with Cancer. J Cancer Educ . 2016;31(4):796-803. doi:10.1007/s13187-015-0946-5

9. Mikan F, Wada M, Yamada M, et al. The Association Between Pain and Quality of Life for Patients With Cancer in an Outpatient Clinic, an Inpatient Oncology Ward, and Inpatient Palliative Care Units. Am J Hosp Palliat Med . 2016;33(8):782-790. doi:10.1177/1049909116630266

10. Marec-Bérard P, Delafosse C, Foussat C. [Cancer-related bone pain in children]. Arch Pediatr Organe Off Soc Francaise Pediatr . 2005;12(2):191-198. doi:10.1016/j.arcped.2004.11.026

11. Caraceni A, Portenoy RK. An international survey of cancer pain characteristics and syndromes. IASP Task Force on Cancer Pain. International Association for the Study of Pain. Pain . 1999;82(3):263-274.

12. Widhe B, Widhe T. Initial symptoms and clinical features in osteosarcoma and Ewing sarcoma. $J$ Bone Joint Surg Am . 2000;82(5):667-674.

13. Ottaviani G, Jaffe N. The Epidemiology of Osteosarcoma. In: Jaffe N, Bruland OS, Bielack S, eds. Pediatric and Adolescent Osteosarcoma . Vol 152. Springer US; 2009:3-13. doi:10.1007/978-1-4419-0284-9_1

14. Zernikow B, Szybalski K, Hübner-Möhler B, et al. Specialized pediatric palliative care services for children dying from cancer: A repeated cohort study on the developments of symptom management and quality of care over a 10-year period. Palliat Med . 2019;33(3):381-391. doi:10.1177/0269216318818022

15. Madden K, Magno Charone M, Mills S, et al. Systematic Symptom Reporting by Pediatric Palliative Care Patients with Cancer: A Preliminary Report. J Palliat Med . Published online February 13, 2019. doi:10.1089/jpm.2018.0545

16. Deandrea S, Montanari M, Moja L, Apolone G. Prevalence of undertreatment in cancer pain. A review of published literature.Ann Oncol Off J Eur Soc Med Oncol . 2008;19(12):1985-1991. doi:10.1093/annonc/mdn419

17. Cohen MZ, Easley MK, Ellis C, et al. Cancer Pain Management and the JCAHO's Pain Standards. $J$ Pain Symptom Manage . 2003;25(6):519-527. doi:10.1016/S0885-3924(03)00068-X

18. WHO Guidelines for the Pharmacological and Radiotherapeutic Management of Cancer Pain in Adults and Adolescents . World Health Organization; 2018. Accessed March 19, 2019. http://www.ncbi.nlm.nih.gov/books/NBK537492/

19. Maltoni M. Opioids, pain, and fear. Ann Oncol . 2008;19(1):5-7. doi:10.1093/annonc/mdm555

20. Jackson TM, Bittman M, Granowetter L. Pediatric Malignant Bone Tumors: A Review and Update on Current Challenges, and Emerging Drug Targets. Curr Probl Pediatr Adolesc Health Care . 2016;46(7):213228. doi:10.1016/j.cppeds.2016.04.002

21. Senerchia AA, Macedo CR, Ferman S, et al. Results of a randomized, prospective clinical trial evaluating metronomic chemotherapy in nonmetastatic patients with high-grade, operable osteosarcomas of the extremities: A report from the Latin American Group of Osteosarcoma Treatment: Metronomic Therapy in Osteosarcoma Patients. Cancer . 2017;123(6):1003-1010. doi:10.1002/cncr.30411

22. On behalf of the Brazilian Collaborative Study Group of Ewing Family of Tumors - EWING1 and the Brazilian Society of Pediatric Oncology - SOBOPE, Becker RG, Gregianin LJ, et al. What is the impact of local control in Ewing sarcoma: analysis of the first Brazilian collaborative study group - EWING1. BMC Cancer . 2017;17(1). doi:10.1186/s12885-017-3391-5 
23. Eleutério SJP, Senerchia AA, Almeida MT, et al. Osteosarcoma in patients younger than 12 years old without metastases have similar prognosis as adolescent and young adults: Osteosarcoma in Children and AYA. Pediatr Blood Cancer . 2015;62(7):1209-1213. doi:10.1002/pbc.25459

24. Wong DL, Baker CM. Pain in children: comparison of assessment scales. Pediatr Nurs . 1988;14(1):9-17.

25. Araujo LC de, Romero B. Pain: evaluation of the fifth vital sign. A theoretical reflection. Rev Dor . 2015;16(4). doi:10.5935/1806-0013.20150060

26. Bieri D, Reeve RA, Champion GD, Addicoat L, Ziegler JB. The Faces Pain Scale for the self-assessment of the severity of pain experienced by children: development, initial validation, and preliminary investigation for ratio scale properties. Pain . 1990;41(2):139-150.

27. Snaman JM, Baker JN, Ehrentraut JH, Anghelescu DL. Pediatric Oncology: Managing Pain at the End of Life. Pediatr Drugs . 2016;18(3):161-180. doi:10.1007/s40272-016-0168-2

28. Jacob J, Matharu JK, Palat G, et al. End-of-Life Treatments in Pediatric Patients at a Government Tertiary Cancer Center in India.J Palliat Med . 2018;21(7):907-912. doi:10.1089/jpm.2017.0632

29. Chi N-C, Demiris G. Family Caregivers' Pain Management in End-of-Life Care: A Systematic Review. Am J Hosp Palliat Med . 2017;34(5):470-485. doi:10.1177/1049909116637359

30. Pakos EE, Nearchou AD, Grimer RJ, et al. Prognostic factors and outcomes for osteosarcoma: an international collaboration. Eur J Cancer Oxf Engl 1990 . 2009;45(13):2367-2375. doi:10.1016/j.ejca.2009.03.005

31. Petrilli AS, Brunetto AL, Cypriano MDS, et al. Fifteen Years' Experience of the Brazilian Osteosarcoma Treatment Group (BOTG): A Contribution from an Emerging Country. J Adolesc Young Adult Oncol . 2013;2(4):145-152. doi:10.1089/jayao.2013.0012

32. Brunetto AL, Castillo LA, Petrilli AS, et al. Carboplatin in the treatment of Ewing sarcoma: Results of the first Brazilian Collaborative Study Group for Ewing Sarcoma Family Tumors-EWING1: Carboplatin in the Treatment of Ewing Sarcoma. Pediatr Blood Cancer . 2015;62(10):1747-1753. doi:10.1002/pbc.25562

33. Kreyer J, Ranft A, Timmermann B, et al. Impact of the Interdisciplinary Tumor Board of the Cooperative Ewing Sarcoma Study Group on local therapy and overall survival of Ewing sarcoma patients after induction therapy. Pediatr Blood Cancer . 2018;65(12):e27384. doi:10.1002/pbc.27384

34. Spraker-Perlman HL, Barkauskas DA, Krailo MD, et al. Factors influencing survival after recurrence in osteosarcoma: A report from the Children's Oncology Group. Pediatr Blood Cancer . 2019;66(1):e27444. doi:10.1002/pbc. 27444

35. Fernández Urtubia B, Trevigno Bravo A, Rodríguez Zamora N, Palma Torres C, Cid Barria L. Uso de opiáceos en niños con cáncer avanzado en cuidados paliativos. Rev Chil Pediatría . 2016;87(2):96-101. doi:10.1016/j.rchipe.2015.10.006

36. Weltgesundheitsorganisation, ed. WHO Guidelines on the Pharmacological Treatment of Persisting Pain in Children with Medical Illnesses . World Health Organization; 2012.

37. Friedrichsdorf S, Postier A. Management of breakthrough pain in children with cancer. J Pain Res . Published online March 2014:117. doi:10.2147/JPR.S58862

38. Friedrichsdorf SJ. Pain Management in Children with Advanced Cancer and During End-of-life Care. Pediatr Hematol Oncol . 2010;27(4):257-261. doi:10.3109/08880011003663416

39. Anghelescu DL, Tesney JM. Neuropathic Pain in Pediatric Oncology: A Clinical Decision Algorithm. Pediatr Drugs . 2019;21(2):59-70. doi:10.1007/s40272-018-00324-4

40. Petrilli AS, de Camargo B, Filho VO, et al. Results of the Brazilian Osteosarcoma Treatment Group Studies III and IV: prognostic factors and impact on survival. J Clin Oncol Off J Am Soc Clin Oncol . 2006;24(7):1161-1168. doi:10.1200/JCO.2005.03.5352 
41. Heinemann M, Ranft A, Langer T, et al. Recurrence of Ewing sarcoma: Is detection by imaging follow-up protocol associated with survival advantage? Pediatr Blood Cancer . 2018;65(7):e27011. doi:10.1002/pbc.27011

42. Reed DR, Hayashi M, Wagner L, et al. Treatment pathway of bone sarcoma in children, adolescents, and young adults. Cancer . 2017;123(12):2206-2218. doi:10.1002/cncr.30589

43. Palermo TM, Chambers CT. Parent and family factors in pediatric chronic pain and disability: An integrative approach: Pain . 2005;119(1-3):1-4. doi:10.1016/j.pain.2005.10.027

\section{Hosted file}

Table 1.docx available at https://authorea.com/users/417398/articles/524528-self-assessmentpain-control-in-end-of-life-children-with-bone-tumors-a-cohort-study

\section{Hosted file}

Table 2.docx available at https://authorea.com/users/417398/articles/524528-self-assessmentpain-control-in-end-of-life-children-with-bone-tumors-a-cohort-study

\section{Hosted file}

Table 3.docx available at https://authorea.com/users/417398/articles/524528-self-assessmentpain-control-in-end-of-life-children-with-bone-tumors-a-cohort-study

\section{Hosted file}

Table 4.docx available at https://authorea.com/users/417398/articles/524528-self-assessmentpain-control-in-end-of-life-children-with-bone-tumors-a-cohort-study

\section{Hosted file}

Table 5.docx available at https://authorea.com/users/417398/articles/524528-self-assessmentpain-control-in-end-of-life-children-with-bone-tumors-a-cohort-study

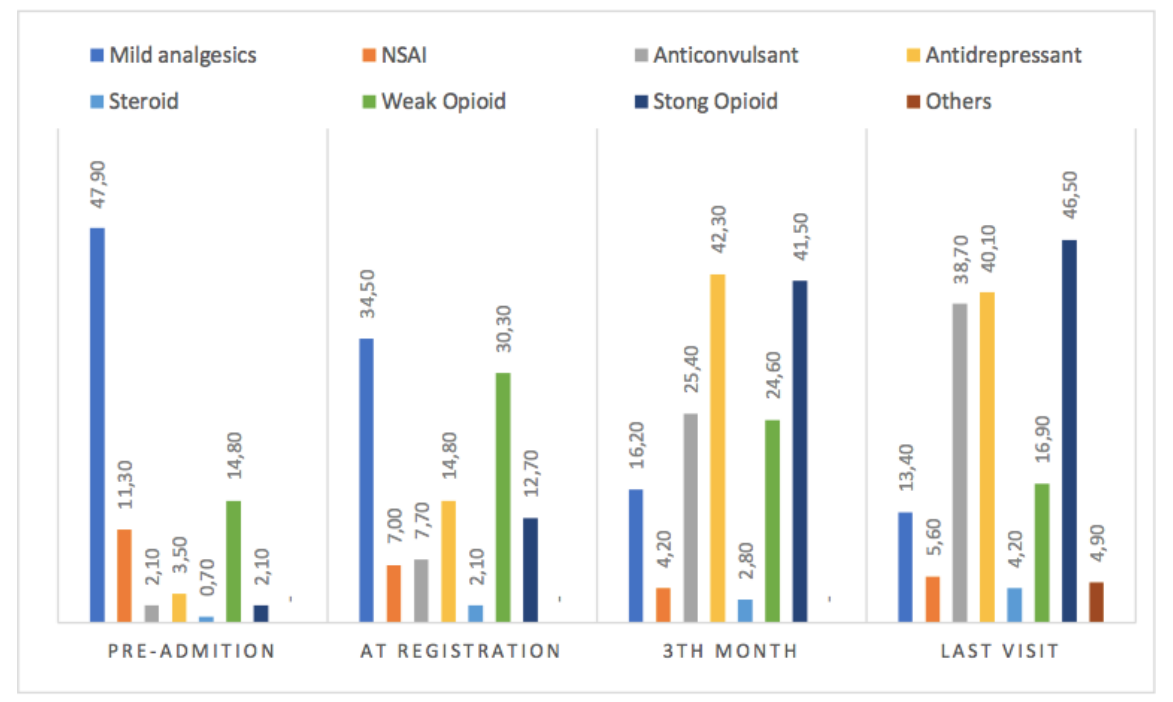

